\title{
Minimally invasive spine surgery decreases postoperative pain and inflammation for patients with lumbar spinal stenosis
}

\author{
HUI PENG ${ }^{1 *}$, GUANGPING TANG $^{2 *}$, XIAOQIANG ZHUANG ${ }^{1}$, SHENGLIN LU $^{1}$, YU BAI $^{1}$ and LI XU ${ }^{3}$ \\ ${ }^{1}$ Department of Orthopedics, Affiliated National Hospital of Guangxi Medical University, Nanning, Guangxi 530021; \\ ${ }^{2}$ Department of Orthopedics, Wuhan Hanyang Hospital, Wuhan, Hubei 430050; \\ ${ }^{3}$ Department of Traumatology, Wuhan No. 1 Hospital, Wuhan, Hubei 430022, P.R. China
}

Received June 6, 2017; Accepted July 6, 2018

DOI: $10.3892 / \mathrm{etm} .2019 .7917$

\begin{abstract}
In certain cases, lumbar spinal stenosis may lead to lumbar nervous disorder. A previous study indicated that minimally invasive spine surgery (MISS) presents benefits compared with conventional open surgery (COS). In the current study, the efficacy of MISS and COS for lumbar spinal stenosis patients was investigated. A total of 82 patients with lumbar spinal stenosis were enrolled and divided into two age-matched groups that received MISS $(n=41)$ or COS $(n=41)$. Patient parameters, including symptoms, inflammatory score, visual analog score (VAS), wound length, Oswestry Disability Index (ODI), hospital stay and postoperative outcomes were analyzed in the current study. Outcomes indicated that both MISS and COS significantly improved symptoms of lumbar spinal stenosis compared with the baseline. It was observed that MISS resulted in decreased wound length and hospital stay compared with COS for patients with lumbar spinal stenosis. The results revealed that MISS had better outcomes compared with COS in improving ODI and inflammatory score for patients with lumbar spinal stenosis. Notably, it was identified that MISS exhibited improved VAS for back and leg pain compared with the COS group for patients with lumbar spinal stenosis. In conclusion, these outcomes indicate that MISS was more effective compared with COS for improving symptoms in patients with lumbar stenosis.
\end{abstract}

\section{Introduction}

Lumbar spinal stenosis is one of the most common causes of lumbar disease and frequently leads to back and waist pain (1). As the elderly population increases, the incidence of lumbar

Correspondence to: Professor Li Xu, Department of Traumatology, Wuhan No. 1 Hospital, 125 Zhongshanda Road, Wuhan, Hubei 430022, P.R. China

E-mail: xuliprofessor@sina.com

*Contributed equally

Key words: lumbar spinal stenosis, minimally invasive spine surgery, visual analog scale, Oswetry Disability Index, inflammatory score spinal stenosis is increasing worldwide (2). Conventional open surgery (COS) has previously been regarded as the gold standard for the treatment of patients with lumbar spinal stenosis (3). Currently, various minimally invasive spine surgery (MISS) techniques are being explored as alternative methods for the treatment of lumbar spinal stenosis (4). MISS has been widely used for the treatment of lumbar spinal stenosis as it is considered to be superior to the COS approach $(5,6)$. The major advantages of MISS are the reduction of infection, unnecessary exposure and tissue trauma (7). The current study compared clinical outcomes in patients with lumbar spinal stenosis who received MISS or COS during a 12-month period.

Postoperative pain is one of the most common characteristics for patients with lumbar spinal stenosis following surgery (8). Postoperative inflammation is still regarded as a crucial criterion for evaluating the efficacy of surgery for patients with lumbar spinal stenosis (9). Retrospective, demographic and clinical investigations have indicated the bacterial causes of postoperative infection in lumbar spinal stenosis patients following surgery (10). Reports have indicated that MISS leads to a decrease in the incidence of infections compared with COS for patients with lumbar spinal stenosis due to the smaller incision and reduced vertebral muscle damage $(11,12)$. However, systemic investigations comparing the outcomes of MISS and COS have not been performed for patients with lumbar spinal stenosis.

In the current study, the efficacy of MISS surgery was investigated for patients with lumbar spinal stenosis. Significant differences between the MISS and COS groups were identified for inflammation score, wound length, hospital stay, Oswestry Disability Index (ODI) and visual analog scale (VAS) score for back and leg pain in a 12-month period following surgery. The current findings suggest that MISS management provides a better therapeutic strategy for patients with lumbar spinal stenosis.

\section{Materials and methods}

Study design, subjects and sampling. A total of 82 patients with L2/3 lumbar spinal stenosis (mean age, 62 years; range, 49.2-68.4 years) were recruited from the Department of Orthopedics at Affiliated National Hospital of Guangxi 
Medical University (Nanning, China) in this retrospective study. The study included 40 male and 42 female patients. Institutional Review Board approval (Affiliated National Hospital of Guangxi Medical University) was obtained for the current study. The study protocol was performed from May 2013 to June 2014 and was approved by the Central Ethics Committee (Ethics Committee of Wuhan No. 1 Hospital, Wuhan, China; approval no. WHHOP20130214). All patients were required to provide written informed consent. The subsequent inclusion criteria included pathological characteristics of lumbar spinal stenosis, non-irritability for ampicillin, surgery history of lumbar stenosis, leg and/or back pain. All patients underwent imaging examination that exhibited lumbar lateral recess stenosis consistent with the symptoms and signs of degenerative lumbar scoliosis.

Drug administration. In total, 82 patients were administered with an antibiotics regimen (ampicillin; Tianjin Tianshili Medicine Co., Ltd., Tianjin, China) in a 14-day continuation phase after surgery. The indicated dosage of resveratrol and/or antibiotic regimen (ampicillin) (both from Sigma-Aldrich; Merck KGaA, Darmstadt, Germany) was 4.0 g/day daily.

Preoperative assessment. All patients received neurological and clinical evaluation prior to surgery (13). The back and leg pain in patients with lumbar spinal stenosis was measured for the lower back and legs using a self-assessment 10-point VAS method (14). Intensity of pain was measured using VAS scores from 0 to 10 ( 0 is no pain and 10 is worst pain). VAS score was measured at $0,3,6,9$ and 12 months following surgery. Disability was assessed using the ODI as described previously (15).

Inflammation severity score. The primary efficacy criterion was the reduction in inflammation severity score in patients with lumbar spinal stenosis following MISS or COS. Inflammation severity score was assessed on day $0,6,12$, 18, 24 and 30 following MISS or COS. Mean inflammation severity score was evaluated as described previously (16).

Surgical procedures. All patients with lumbar spinal stenosis received MISS $(\mathrm{n}=41)$ and $\operatorname{COS}(\mathrm{n}=41$; allocated at random) following administration of general anesthesia in the prone position. The MISS and COS surgery was performed in a consistent way and an operative microscope was used in all cases (17).

In the MISS group, a C-arm X-ray machine (Biplanar 500e; Swemac Medical Appliances AB, Täby, Sweden), METRx Quadrant System and percutaneous pedicle screw (both from Medtronic, Minneapolis, MN, USA) were prepared. An incision was planned by connecting a line between the outer portions of both end pedicles ( $3.0 \mathrm{~cm}$ off midline). Then, a skin incision of 3-4 $\mathrm{cm}$ was made on the more symptomatic side or more severe pathology side according to imaging. Decompression was conducted by cutting the inferior portion of the lamina, hypertrophied superior and inferior articular processes, and ligamenta flava. Then, the intervertebral space was enlarged with a distractor followed by a PEEK cage (Capstone; Medtronic). Then, percutaneous pedicle screw fixation was conducted.
For the COS group, following routine disinfection and draping, a G-arm machine was used to confirm the affected segment. Then, a longitudinal incision was made in the middle of the spine, and muscular fasciae were cut apart. Musculus sacrospinalis were then bluntly dissected until the lumbar transverse process was exposed. Pedicle screws were placed into the upper and subjacent vertebral pedicle of the segmental lesions. Spinous process, lamina, hyperplasia of yellow ligament and interior zygapophysis were removed according to the scope of the lesions, and lateral recess as well as nerve root canal was enlarged with the protection of dural sac and nerve tissue. Then, fibrous rings were cut and nucleus pulposus was removed, and the intervertebral space was opened. The removed laminar and zygapophysis were crushed into smaller pieces for incorporation as autograft, then the cage with crushed bones was also inserted. Next, titanium rods were used to connect the screws and fixed. Finally, negative pressure drainage was placed and the incision was sewn up.

Outcome assessment. ODI score and VAS score in lumbar spinal stenosis patients was logged at 0,3,6,9 and 12 months following MISS or COS (18). Patient satisfaction following MISS surgery was analyzed using the patient satisfaction index (PSI, a modified sub-item of the North American Spine Society outcome questionnaire) method, as reported previously (19).

Statistical analysis. Continuous variables are reported as the mean \pm standard deviation. All data were analyzed using SPSS Statistics 19.0 (IBM Corp., Armonk, NY, USA) and GraphPad Prism 5.0 (GraphPad Software, Inc., La Jolla, CA, USA) with the help of Microsoft Excel 2010 (Microsoft Corporation, Redmond, WA, USA). Unpaired data were compared by Student's t-test and comparisons between multiple groups were performed using one-way analysis of variance (ANOVA) followed by Tukey's post hoc test. VAS scores exhibited normal distribution prior to surgery and were analyzed by the Wilcoxon test prior to and following surgery. The t-test was used to analyze ODI index and inflammation score prior to and following surgery. $\mathrm{P}<0.05$ was considered to indicate a statistically significant difference.

\section{Results}

Characteristics of lumbar spinal stenosis patients. A total of 82 patients with lumbar spinal stenosis were enrolled in the current study. It was demonstrated that the mean body mass index was 26.4 and $25.2 \mathrm{~kg} / \mathrm{m}^{2}$ in the MISS and COS groups, respectively. There was no notable difference in the incidence of leg and back pain between the MISS and COS groups. The characteristics and symptoms of lumbar spinal stenosis patients are summarized in Table I. It was observed that there were no significant differences between patients in the MISS and $\operatorname{COS}$ groups prior to surgery.

Efficacy of MISS on postoperative pain for patients with lumbar spinal stenosis. As indicated in Fig. 1, patients in the MISS group exhibited lower VAS scores compared with the $\operatorname{COS}$ group at 3 and 6 months post-surgery. There was no 
Table I. Characteristics of patients with lumbar spinal stenosis.

\begin{tabular}{lcc}
\hline Parameter & MISS & COS \\
\hline Total number & 41 & 41 \\
Male & 19 & 21 \\
Female & 22 & 20 \\
Age (years) & $50.4-65.6$ & $49.2-68.4$ \\
Mean BMI $\left(\mathrm{kg} / \mathrm{m}^{2}\right)$ & 26.4 & 25.2 \\
Neurogenic claudication & 17 & 14 \\
Numbness & 26 & 29 \\
Leg pain & 24 & 20 \\
Back pain & 17 & 21 \\
Follow-up duration (months) & 12 & 12
\end{tabular}

MISS, minimally invasive spine surgery; COS, conventional open surgery; BMI, body mass index.

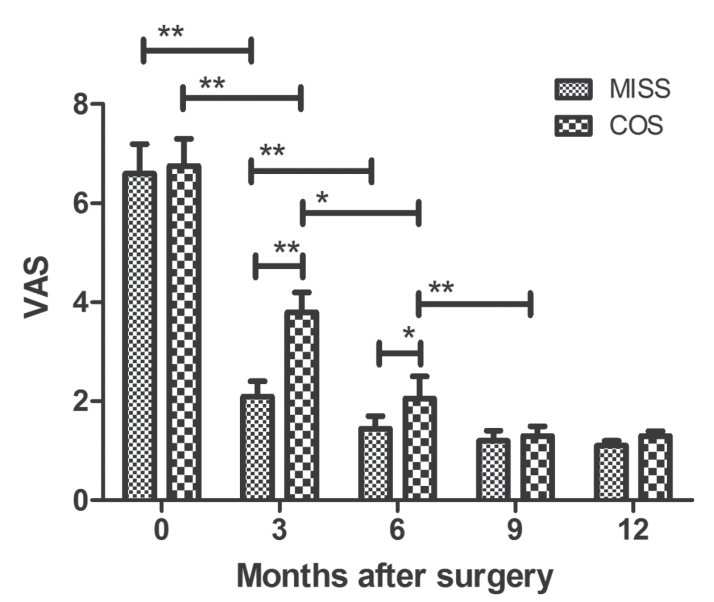

Figure 1. Effects of MISS and COS in relieving postoperative pain determined by VAS score. MISS, minimally invasive spine surgery; COS, conventional open surgery; VAS, visual analog scale. ${ }^{*} \mathrm{P}<0.05,{ }^{* *} \mathrm{P}<0.01$.

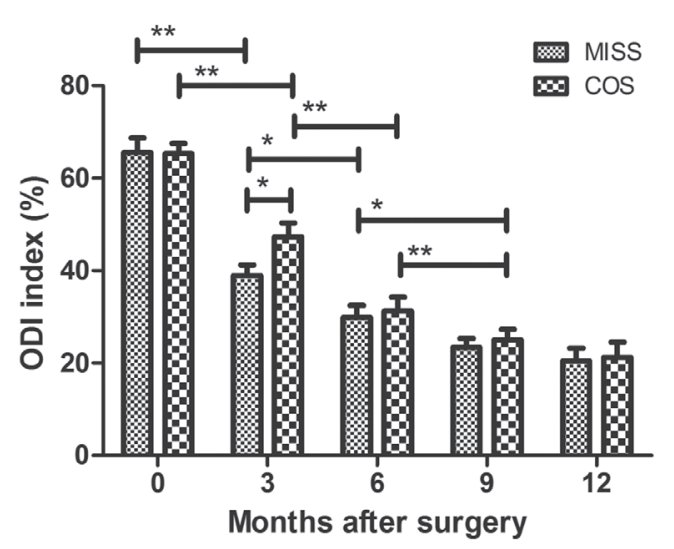

Figure 2. Effects of MISS and COS in relieving postoperative pain determined by ODI. MISS, minimally invasive spine surgery; COS, conventional open surgery; ODI, Oswetry Disability Index. ${ }^{*} \mathrm{P}<0.05,{ }^{* *} \mathrm{P}<0.01$.

significant difference in VAS between the two groups at 9 or 12 months post-surgery. As indicated in Fig. 2, MISS exhibited greater efficacy compared with COS at relieving back and leg

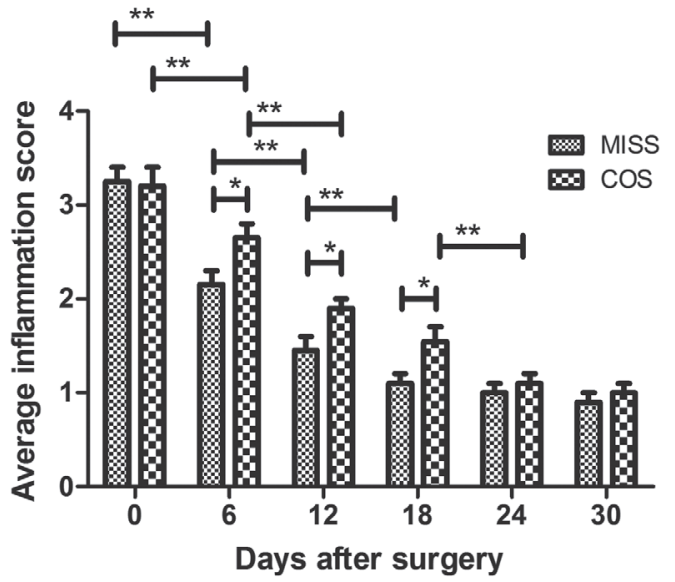

Figure 3. Effects of MISS and COS on inflammatory score for patients with lumbar spinal stenosis. MISS, minimally invasive spine surgery; COS, conventional open surgery. ${ }^{*} \mathrm{P}<0.05,{ }^{* *} \mathrm{P}<0.01$.

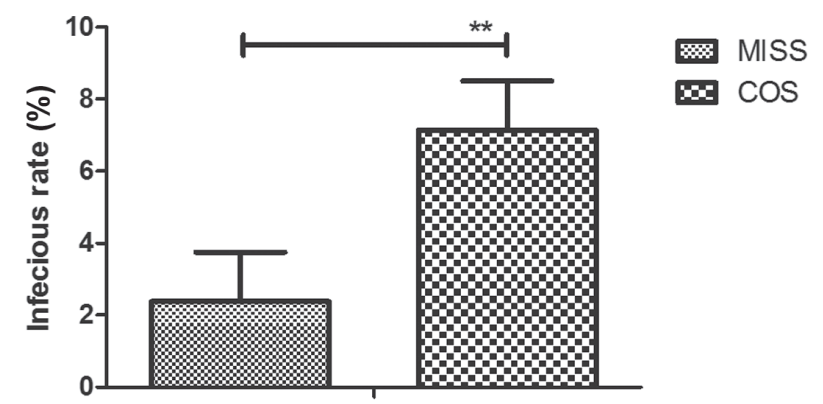

Figure 4. Effects of MISS and COS on infection rates in patients with lumbar spinal stenosis. MISS, minimally invasive spine surgery; COS, conventional open surgery. ${ }^{* *} \mathrm{P}<0.01$

pain at 3 months post-surgery for patients with lumbar spinal stenosis. None of the patients demonstrated postoperative deterioration in neurological status.

Efficacy of MISS for postoperative inflammation and infection in patients with lumbar spinal stenosis. Inflammation and infection was analyzed in patients with lumbar spinal stenosis following MISS or COS. All patients received ampicillin antibiotic regimen for a total of 14 days. The inflammatory score was significantly decreased in patients who received MISS compared with those who received COS on days 6,12 and 18 post-surgery (Fig. 3). As indicated in Fig. 4, patients who received MISS exhibited significantly lower infection rates compared with $\operatorname{COS}(2.38$ vs. $7.17 \%)$. None of the patients exhibited postoperative incurable infection.

Efficacy of MISS for recovery in patients with lumbar spinal stenosis. As indicated in Fig. 5, preoperative wound length was significantly increased in the COS group compared with the MISS group (4.04 vs. $1.32 \mathrm{~cm})$. Length of hospital stay was significantly shorter in the MISS group compared with the COS group (Fig. 6; 6.5 vs. 12 days). Patient satisfaction was significantly higher in the MISS group (94.8\%) compared with the COS group (80.3\%), as determined by PSI (overall satisfaction) during the 12-month follow-up period (Fig. 7). None of the patients succumbed during the 12-month follow-up period. 


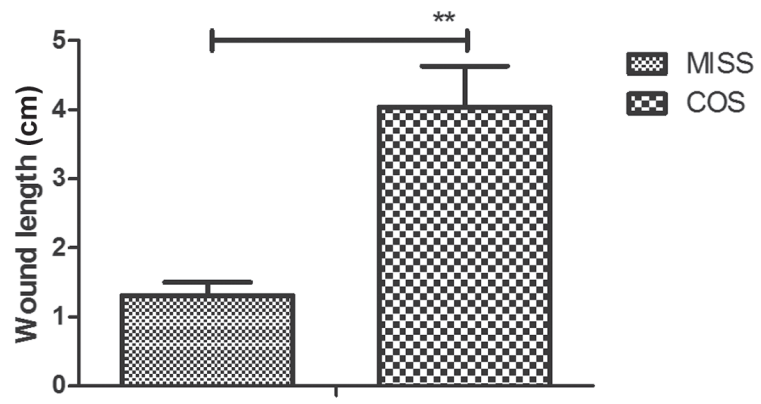

Figure 5. Effects of MISS and COS on wound length in patients with lumbar spinal stenosis. MISS, minimally invasive spine surgery; COS, conventional open surgery. ${ }^{* *} \mathrm{P}<0.01$.

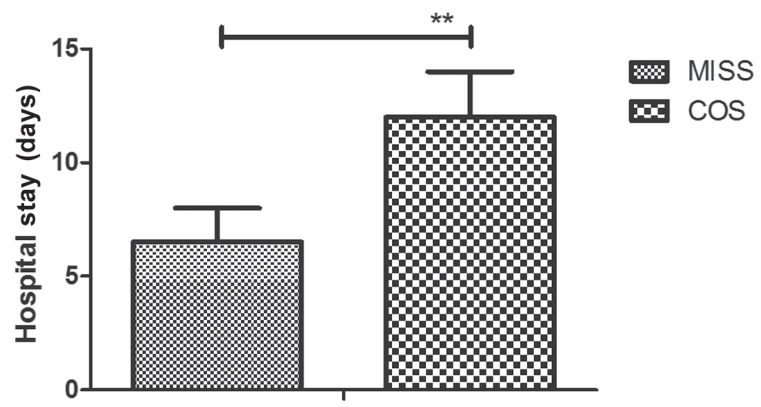

Figure 6. Effects of MISS and COS on length of hospital stay for patients with lumbar spinal stenosis. MISS, minimally invasive spine surgery; COS, conventional open surgery. ${ }^{* *} \mathrm{P}<0.01$.

\section{Discussion}

Lumbar spinal stenosis is one of the most common orthopedic diseases and the incidence rate is more than 5\% in the elderly population worldwide (20). COS is the most common method used for the treatment of lumbar spinal stenosis (18). However, in recent years, MISS has been widely used for patients with lumbar spinal stenosis (21). In the current study, the efficacy of MISS and COS was compared in patients with lumbar spinal stenosis. It was identified that MISS presented advantages over COS for patients with lumbar spinal stenosis, including improvements of back and leg pain, postoperative inflammation and infection rate in the clinic. The current findings indicated that patients with lumbar spinal stenosis who received MISS reported higher satisfaction compared with those who received COS during a 12-month follow-up period. None of the patients demonstrated postoperative incurable infection, postoperative deterioration in neurological status or mortality.

Complications occur frequently for patients with lumbar spinal stenosis following surgery, including surgical site infection, bone graft migration, intraoperative neurological injury and dural tear (22). A previous study indicated that microendoscopic discectomy MISS techniques could decrease postoperative complications for lumbar spinal stenosis patients (23). In the current study, no cases of intraoperative neurological injury, bone graft migration or dural tear were observed. However, 4 patients in the COS group presented with surgical site infection, while 1 patient presented with surgical site infection in the MISS group. Notably, MISS exhibited better therapeutic effects compared with COS in

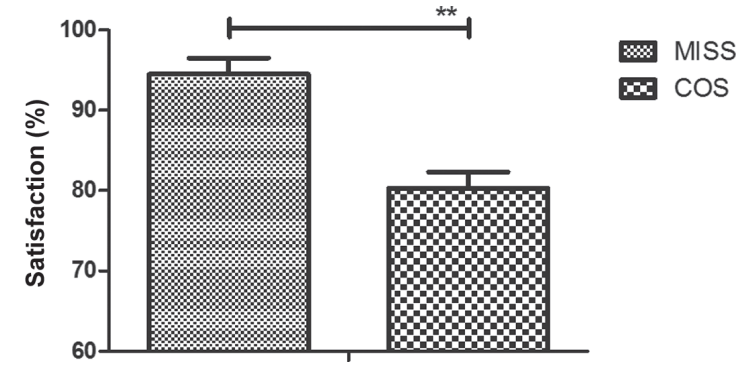

Figure 7. Effects of MISS and COS on patient satisfaction as determined by PSI (overall satisfaction) during the 12-month follow-up period. MISS, minimally invasive spine surgery; COS, conventional open surgery; PSI, patient satisfaction index. ${ }^{* *} \mathrm{P}<0.01$.

relieving back and leg pain at 0 and 3 months post-surgery as determined by ODI and VAS.

For patients with lumbar spinal stenosis, inflammation may affect the length of hospital stays (24). A previous study reviewed the role of muscular markers, inflammatory parameters and cytokines and concluded that MISS efficiently decreased post-surgery inflammation compared with COS (24). In the current study, it was observed that patients with lumbar spinal stenosis that underwent MISS exhibited a lower inflammation score and shorter hospital stays compared with those that underwent COS. A previous study indicated that decreasing postoperative surgical infection using MISS may decrease mortality rates (10). In the current study, it was reported that MISS efficiently decreased postoperative surgical infections in patients with lumbar spinal stenosis compared with COS.

In conclusion, the current findings indicate that are significant differences between the outcomes of MISS and COS for patients with lumbar spinal stenosis in terms of inflammation score, infection rate, VAS and ODI. The study identified that MISS resulted in improved symptoms in patients with lumbar stenosis, including reduced postoperative pain and shorter hospital stays. Therefore, it could be regarded as a standard treatment for patients with lumbar spinal stenosis. However, prospective randomized studies should be performed in a larger lumbar spinal stenosis population in order to verify the conclusions of the current study.

\section{Acknowledgements}

Not applicable.

\section{Funding}

No funding was received.

\section{Availability of data and materials}

The datasets used and/or analyzed during the current study are available from the corresponding author on reasonable request.

\section{Authors' contributions}

HP performed the experiments. GT, XZ, SL and YB prepared and analyzed experimental data. LX designed the experiments. 


\section{Ethics approval and consent to participate}

The present study was approved by the Ethics Committee of Affiliated National Hospital of Guangxi Medical University (Nanning, China). Written informed consent was obtained from all patients.

\section{Patient consent for publication}

Written informed consent was obtained from all patients.

\section{Competing interests}

The authors declare that they have no competing interests.

\section{References}

1. Houten JK and Nasser R: Symptomatic progression of degenerative scoliosis after decompression and limited fusion surgery for lumbar spinal stenosis. J Clin Neurosci 20: 613-615, 2013.

2. Kuang MJ, Ma JX and Ma XL: Decompression and coflex interlaminar stabilization compared with conventional surgical procedures for lumbar spinal stenosis: A systematic review and meta-analysis. Int J Surg 45: 164-165, 2017.

3. Ulrich NH, Kleinstück F, Woernle CM, Antoniadis A, Winklhofer S, Burgstaller JM, Farshad M, Oberle J, Porchet F and Min K; LumbSten Research Collaboration: Clinical outcome in lumbar decompression surgery for spinal canal stenosis in the aged population: A prospective Swiss multicenter cohort study. Spine (Phila Pa 1976) 40: 415-422, 2015.

4. Minamide A, Yoshida M, Iwahashi H, Simpson AK, Yamada H, Hashizume H, Nakagawa Y, Iwasaki H, Tsutsui S, Kagotani R, et al Minimally invasive decompression surgery for lumbar spinal stenosis with degenerative scoliosis: Predictive factors of radiographic and clinical outcomes. J Orthop Sci 22: 377-383, 2017.

5. Moojen WA and Peul WC: Minimally invasive surgery for lumbar spinal stenosis. BMJ 350: h1664, 2015.

6. Kim HJ, Lee JI, Kang KT, Chang BS, Lee CK, Ruscheweyh R, Kang SS and Yeom JS: Influence of pain sensitivity on surgical outcomes after lumbar spine surgery in patients with lumbar spinal stenosis. Spine (Phila Pa 1976) 40: 193-200, 2015.

7. Vasudeva VS and Chi JH: Fusion surgery for lumbar spinal stenosis. N Engl J Med 375: 598, 2016.

8. Park CK, Kim SB, Kim MK, Park BJ, Choi SG, Lim YJ and Kim TS: Comparison of treatment methods in lumbar spinal stenosis for geriatric patient: Nerve block versus radiofrequency neurotomy versus spinal surgery. Korean J Spine 11: 97-102, 2014.

9. Wang ZH, Wu DH, Ma C and Dai WX: Surgical treatment of postoperative deep wound infection after posterior lumbar interlumbar fusion of the lumbar stenosis. Zhongguo Gu Shang 25: 928-930, 2012 (In Chinese).

10. Yaldiz C, Yaldiz M, Ceylan N, Kacira OK, Ceylan D, Kacira T, Kizilcay $\mathrm{G}$ and Tanriverdi T: Retrospective, demographic, and clinical investigation of the causes of postoperative infection in patients with lumbar spinal stenosis who underwent posterior stabilization. Medicine (Baltimore) 94: e1177, 2015.
11. Polikandriotis JA, Hudak EM and Perry MW: Minimally invasive surgery through endoscopic laminotomy and foraminotomy for the treatment of lumbar spinal stenosis. J Orthop 10: 13-16, 2013.

12. Lauryssen C: Technical advances in minimally invasive surgery: Direct decompression for lumbar spinal stenosis. Spine (Phila Pa 1976) 35 (Suppl 26): S287-S293, 2010.

13. Sakalauskienè G, Obelienius V, Pilvinienė R and Jauniškienè D: Evaluation of daily outpatient multidisciplinary rehabilitative treatment of patients with musculoskeletal, neurological and traumatic disorders in a municipality outpatient setting. Medicina (Kaunas) 52: 61-68, 2016.

14. Scott J and Huskisson EC: Graphic representation of pain. Pain 2: 175-184, 1976.

15. Copay AG, Glassman SD, Subach BR, Berven S, Schuler TC and Carreon LY: Minimum clinically important difference in lumbar spine surgery patients: A choice of methods using the Oswestry disability index, Medical outcomes study questionnaire Short Form 36, and pain scales. Spine J 8: 968-974, 2008.

16. Lane SS and Holland EJ: Loteprednol etabonate $0.5 \%$ versus prednisolone acetate $1.0 \%$ for the treatment of inflammation after cataract surgery. J Cataract Refract Surg 39: 168-173, 2013.

17. Hudak EM and Perry MW: Outpatient minimally invasive spine surgery using endoscopy for the treatment of lumbar spinal stenosis among obese patients. J Orthop 12: 156-159, 2015.

18. Haddadi K and Ganjeh Qazvini HR: Outcome after surgery of lumbar spinal stenosis: A randomized comparison of bilateral laminotomy, trumpet laminectomy, and conventional laminectomy. Front Surg 3: 19, 2016.

19. Aghayev E, Elfering A, Schizas C and Mannion AF; SWISSSpine Registry Group: Factor analysis of the North American Spine Society outcome assessment instrument: A study based on a spine registry of patients treated with lumbar and cervical disc arthroplasty. Spine J 14: 916-924, 2014.

20. Coronado-Zarco R, Cruz-Medina E, Arellano-Hernández A, Chavez-Arias D and León-Hernández SR: Effectiveness of calcitonin in intermittent claudication treatment of patients with lumbar spinal stenosis: A systematic review. Spine (Phila Pa 1976) 34: E818-E822, 2009.

21. Rubino F, Deutsch H, Pamoukian V, Zhu JF, King WA and Gagner M: Minimally invasive spine surgery: An animal model for endoscopic approach to the anterior cervical and upper thoracic spine. J Laparoendosc Adv Surg Tech A 10: 309-313, 2000.

22. Podichetty VK, Spears J, Isaacs RE, Booher J and Biscup RS: Complications associated with minimally invasive decompression for lumbar spinal stenosis. J Spinal Disord Tech 19: 161-166, 2006.

23. Ikuta K, Tono O, Tanaka T, Arima J, Nakano S, Sasaki K and Oga M: Surgical complications of microendoscopic procedures for lumbar spinal stenosis. Minim Invasive Neurosurg 50: 145-149, 2007.

24. Imajo Y, Taguchi T, Neo M, Otani K, Ogata T, Ozawa H, Miyakoshi N, Murakami $\mathrm{H}$ and Iguchi T: Complications of spinal surgery for elderly patients with lumbar spinal stenosis in a super-aging country: An analysis of 8,033 patients. J Orthop Sci 22: 10-15, 2017.

This work is licensed under a Creative Commons Attribution-NonCommercial-NoDerivatives 4.0 International (CC BY-NC-ND 4.0) License. 